\title{
Uso de Álcool por Estudantes de Medicina segundo Características de Cursos e Escolas Médicas: uma Revisão da Literatura
}

\author{
Alcohol use by Medical Students according \\ to Course and Medical School Characteristics: \\ a Literature Review
}

\author{
Maria Isabel do Nascimento ${ }^{\text {एீ }}$ \\ Juliana dos Santos Costa ${ }^{I}$ \\ Marcos André Pereira \\ Meika Santana Kiepper ${ }^{I}$ \\ Natália Braga KeherI \\ Roberta Fernandes Silva e Moraes
}

PALAVRAS-CHAVE

- Álcool.

- Consumo de Bebidas Alcoólicas.

- Estudantes de Medicina.

- Prevalência.

\section{RESUMO}

Introdução: $O$ álcool é fator de risco para várias condições de saúde e responde por mais de 3 milhões de óbitos por ano no mundo. A substância contribui para o desenvolvimento de doenças crônicas, como as cardiovasculares e o câncer, e para condições agudas relacionadas ao efeito direto sobre o sistema nervoso central. Apesar disso, seu uso constituí prática comum entre estudantes, particularmente entre os alunos de Medicina. Os objetivos do presente estudo foram: (i) fazer uma revisão de literatura para identificar estudos de prevalência de uso de álcool entre estudantes de Medicina de escolas brasileiras; (ii) analisar as estimativas de prevalência de uso de álcool segundo características dos cursos e das escolas médicas. Método: Trata-se de revisão da literatura efetuada para resgatar publicações nas bases bibliográficas (i) Literatura Latino-Americana em Ciências de Saúde (Lilacs), utilizando a combinação de descritores de saúde [(estudantes de medicina AND álcool)], e (ii) US National Library of Medicine of National Institute of Health (Medline/Pubmed), usando a combinação de descritores de saúde [(medical students AND alcohol AND Brazil)]. Os padrões de uso de álcool nos últimos sete dias (7D), 30 dias (30D) e último ano (ANO) foram analisados pela visualização de gráficos de ações e de dispersão, bem como pela verificação de correlação. Resultados: Foram incluídos 14 estudos, dos quais oito foram desenvolvidos na Região Sudeste. A maior parte das instituições envolvidas tinha administração pública $(n=8)$ e estava localizada em capitais $(n=8)$. A prevalência de uso de álcool nos últimos sete dias variou de 23,0\% a 46,5\%; nos últimos 30 dias, variou de 20,2\% a 87,6\%; e no último ano, variou de 79,3\% a 92,9\%. A correlação da prevalência com a carga horária do curso e com o tempo de existência da escola mostrou intensidade fraca nos três padrões de uso analisados. No padrão de uso de sete dias, a direção da correlação foi negativa nas duas características (carga horária do curso e tempo de existência da escola). Nos últimos 30 dias, a direção da correlação foi negativa com a carga horária e positiva com o tempo de existência da escola. No último ano, a direção da correlação foi positiva com a carga horária e negativa com o tempo de existência da escola. Conclusões: O uso de álcool é tema recorrente na literatura científica no Brasil, e as prevalências de uso nos últimos sete dias, 30 dias e último ano são altas entre estudantes de Medicina. A influência das características de escolas médicas e de cursos de Medicina sobre o uso de substâncias psicoativas carece de mais atenção da comunidade científica, sendo notória a necessidade do envolvimento das instituições de ensino superior no controle do problema do álcool no Brasil. 


\section{KEY-WORDS}

- Alcohol.

- Alcohol Drinking.

- Students, Medical.

- Prevalence.

\begin{abstract}
Introduction: Alcohol is a risk factor for several health conditions and accounts for more than three million deaths per year worldwide. The substance contributes to the development of chronic diseases such as cardiovascular and cancer, as well as for acute conditions related to direct effects on the central nervous system. Despite this, its use is common practice among university students, including medical students. Objectives: The objectives were (i) to develop a literature review to identify studies into the prevalence of alcohol use among students at Brazilian medical schools; and (ii) to analyze the estimates of prevalence according to medical course and school characteristics. Method: Literature review developed to search publications in the (i) Latin American and Caribbean Center in Health Sciences Information (Lilacs) database, using the word combination [(estudantes de medicina AND álcool)] and in the US National Library of Medicine of National Institute of Health (Medline/Pubmed) database, using the word combination [(medical students AND alcohol AND Brazil)]. Alcohol use patterns in the past seven days, past thirty days, and past year were analyzed by plotting and visualization of point estimates and confidence intervals, as well as correlations. Results: Fourteen studies were included in this review. Eight of them were developed in the southeast region. Most institutions were under public administration $(n=8)$, and were in state capitals $(n$ $=8$ ). The prevalence of alcohol use in the past seven days ranged from $23.0 \%$ to $46.5 \%$, in the past thirty days ranged from $20.2 \%$ to $87.6 \%$, and in the past year ranged from $79.3 \%$ to $92.9 \%$. The correlations between prevalence and workload, and between prevalence and age of school showed a weak intensity on the three use patterns analyzed. The correlation directions followed diversified patterns. In the past seven days, the correlation directions were negative both with workload and age of the school. In the past thirty days, the correlation direction was negative with the workload, and positive with the age of the school. In the past year, the correlation direction was positive with workload, and negative with the age of the school. Conclusions: Alcohol use is a recurring theme in Brazilian scientific literature and the estimates of prevalence of its use in the past seven days, in the past thirty days and in the past year are high among medical students. The influence of medical school and the medical course characteristics on the use of psychoactive substances requires more attention from the scientific community, and the involvement of the higher education institutions in controlling the alcohol problem in Brazil is fundamental.
\end{abstract}

\section{INTRODUÇÃO}

O consumo de álcool é prática disseminada na população mundial, sendo recomendado o desenvolvimento de políticas e ações voltadas a proteger pessoas que preferem não beber, permanecendo na condição de abstêmias, bem como a desestimular o consumo entre os usuários de bebidas alcoólicas ${ }^{1}$.

No Brasil, uma das ações de monitoramento de fatores de risco na área de vigilância em saúde coordenadas pelo Ministério da Saúde mostra que o consumo de álcool na população geral, nos últimos 30 dias que antecederam a entrevista, apresenta prevalências mais elevadas entre os brasileiros de maior escolaridade (12 e mais anos de estudo) e nas faixas etárias de 18 a 24 anos e de 25 a 34 anos $^{2}$. Tais resultados reforçam a ideia de que os estudantes universitários, de modo geral, per- tencem ao núcleo da população vulnerável às consequências do consumo do álcool, apesar da existência de políticas implementadas no País.

Um levantamento sobre o uso de álcool por universitários, conduzido nas 27 capitais brasileiras, mostrou que a prevalência de consumo entre eles é consistentemente mais elevada comparando-se com a população geral, tanto para uso na vida $(86,2 \%$ versus $74,6 \%)$, quanto nos últimos 12 meses $(72,0 \%$ versus $49,8 \%$ ), bem como para os últimos 30 dias (60,5\% versus $38,3 \%)^{3}$.

A vida na universidade é preenchida por uma série de novidades que direta ou indiretamente contribui para a experimentação, manutenção e intensificação do uso do álcool ${ }^{4}$. Desde os anos 1990, estudos apontam que a faculdade de 
Medicina é locus de iniciação de consumo de álcool ${ }^{5}$. Contudo, a universidade tem por natureza papel social e compromissos científicos que devem ser desafiados e intensificados a fim de se inibir o uso desta e de outras substâncias psicoativas.

No Brasil, o hábito de ingerir bebida alcoólica se expande a diferentes cursos superiores da área de saúde ${ }^{6}$. No Rio de Janeiro, dados de 1998 que envolveram 1.005 acadêmicos de Medicina de quatro universidades públicas mostraram que o uso de álcool na vida e nos últimos 30 dias alcançou, respectivamente, as proporções de $96,4 \%$ e $58,8 \% 7$.

Considerando a gravidade dos problemas gerados pelo uso do álcool e o enfoque da literatura, que envolve quase exclusivamente as características individuais dos estudantes de graduação, neste estudo postulamos que a prevalência do uso de álcool nos estudantes de Medicina, no Brasil, pode manter relação com características da escola médica, bem como com as do curso de Medicina. Assim, os objetivos deste estudo foram: (i) efetuar uma revisão de literatura para identificar estudos de prevalência de uso de álcool entre estudantes de Medicina de escolas brasileiras; (ii) analisar as estimativas de prevalência de uso de álcool segundo características dos cursos e das escolas médicas.

\section{MÉTODO}

\section{Estratégias e período de busca}

Este estudo abrange uma revisão sistemática da literatura com foco em publicações relativas a estudantes de escolas médicas brasileiras. Essa revisão foi desenvolvida isenta de limitadores de idioma e de período e foi encerrada em março de 2018. Foram utilizadas estratégias de busca com o uso de descritores (DeCs Terminologia) de saúde indexados à base de dados do Centro Latino-Americano e do Caribe de Informações em Ciências da Saúde (Bireme). As bases de dados bibliográficos consultadas foram a de Literatura Latino-Americana em Ciências de Saúde (Lilacs) e da US National Library of Medicine of National Institute of Health (Medline/Pubmed). As combinações de descritores usadas na busca no Lilacs foram [(estudantes de medicina $A N D$ álcool)] e no Pubmed foram [(medical students AND alcohol AND Brazil)]. A busca foi ampliada também à lista de referências dos artigos selecionados para leitura.

\section{Critérios de inclusão}

Os critérios de inclusão considerados foram: (i) artigos originais; (ii) informação clara de identificação da escola onde o estudo foi desenvolvido; (iii) fornecimento de estimativas de prevalência de uso de álcool.

\section{Critérios de exclusão}

Foram excluídos os estudos que envolveram mais de uma escola, mas que não forneceram estimativas de prevalência individualizadas; e estudos conduzidos em instituições cujos dados de interesse da pesquisa não se encontravam disponibilizados em nenhuma fonte online (site da própria instituição, Ministério da Educação e Cultura, etc.).

\section{Formulários e extração dos dados}

Foi desenvolvido um formulário para registrar as informações básicas de identificação dos estudos (autor, ano, periódico, instituição, método de ensino); localização da escola médica (região geográfica e capital/interior); tipo de gestão (pública/ privada); características do curso (tempo de existência e carga horária do curso); prevalência de uso de álcool (PV); quantitativo de estudantes entrevistados $(\mathrm{N})$ e de estudantes usuários de bebidas alcoólicas (n).

Estiveram envolvidos na coleta de dados os alunos de iniciação científica, estudantes de Medicina, coautores do presente manuscrito, supervisionados pela orientadora. Diante da falta de consenso sobre a inclusão ou não de um estudo, a decisão foi tomada pela orientadora.

Os indicadores de uso de álcool foram extraídos segundo o padrão de medição relatado nos estudos, sendo considerado para a presente revisão se o estudante (i) bebeu nos últimos sete dias (7D), (ii) bebeu nos últimos 30 dias (30D) e (iii) bebeu no último ano (ANO).

Com a consulta às bases científicas (Lilacs e Pubmed), 131 publicações foram identificadas. Após a triagem inicial, aplicação dos critérios de inclusão e exclusão, eliminação das repetições e consulta à lista de referências, foram incluídos 14 artigos. O fluxograma síntese da estratégia de busca e seleção de artigos encontra-se na Figura 1.

A qualidade metodológica dos estudos incluídos teve como parâmetro oito critérios, concebidos em 1998 por Loney e colaboradores ${ }^{8}$, com a finalidade de orientar análises de estudos de prevalência e de incidência. Para esses autores, é importante verificar questões a respeito de: (i) cálculo e adequação do tamanho da amostra; (ii) definição dos indivíduos para composição da população de estudo; (iii) processo adotado para seleção dos participantes; (iv) uso de instrumento de aferição validado; (v) coleta e mensuração da informação sem viés; (vi) verificação de taxa de resposta e de recusa; (vii) estimativa de intervalos de confiança; (viii) apresentação de resultados para grupos e subgrupos, com possibilidade de generalização. Critérios adequados recebem escore 1, e o somatório máximo dos escores é de 8 pontos. 




Análise da relação entre indicadores de prevalência e características das escolas médicas

A análise da relação entre os indicadores de prevalência e as características das escolas médicas foi feita primeiramente pela plotagem e visualização de gráficos de ações onde, no eixo do $Y$, constavam as estimativas de prevalência e os limites de intervalos de confiança, e no eixo de $X$, os diferentes estudos. A apresentação dos gráficos foi organizada segundo região geográfica, modelo de gestão e localização na capital ou no interior, considerando os diferentes padrões de mensuração [(7D), (30D), (1ANO)].

\section{Correlação entre indicadores de prevalência e características dos cursos de Medicina}

A correlação entre os indicadores de prevalência e as características dos cursos de Medicina foi feita pela plotagem e visualização de gráficos de dispersão, considerando no eixo do $\mathrm{Y}$ as estimativas de prevalência, e no eixo do $X$ os anos de existência do curso e a carga horária do curso.

O cálculo de anos de existência do curso foi baseado na diferença entre o ano de início das atividades de ensino no curso e o ano em que ocorreu a coleta de dados. A carga horária foi obtida em documentos disponibilizados online pelas universidades ou na página da internet Escolas Médicas ${ }^{9}$ ou em site oficial do Ministério da Educação. Para esta análise, foi usada a carga horária vigente no ano da coleta dos dados da pesquisa.

\section{Análise estatística}

Para verificação de diferenças entre as estimativas de prevalência, considerou-se a comparação de intervalos de confiança de 95\% (IC 95\%). Prevendo-se que tais intervalos poderiam estar ausentes de algumas das publicações, considerou-se a equação $I C(95 \%)=p o \pm 1,96 \sqrt{ }(p o(1-p o)) / n$ para obtenção dos limites superiores e inferiores dos intervalos de confiança de IC 95\%, onde po é a prevalência estimada, e $n$ é a amostra da população incluída no estudo.

\section{Aspectos éticos}

O presente estudo segue as normas recomendadas para pesquisas científicas que envolvem seres humanos e foi desenvolvido com dados já publicados e disponibilizados online em bases de literatura científicas, sem identificação dos sujeitos incluídos nos estudos originais.

\section{RESULTADOS}

Os estudos incluídos $(\mathrm{n}=14)$ nesta revisão foram desenvolvidos na predominantemente Região Sudeste $(n=8)$ e tiveram casuísticas selecionadas após o ano 2000 ( $n=11)$. Oito deles eram de instituições de administração pública e seis de administração privada. O método de ensino principal foi o tradicional. Somente dois periódicos (Revista Brasileira de Psiquiatria e Jornal Brasileiro de Psiquiatria) contribuíram com mais de um artigo. O escore relativo à qualidade metodológica das publicações variou de 3 a 7. As características dos estudos incluídos encontram-se na Tabela 1.

Entre os 14 estudos analisados, apenas um $^{17}$ apresentou resultados de dois anos independentes (1996 e 2001), dez ${ }^{10-}$ 13,15,17,18, 20,22,23 examinaram mais de um indicador (7D e/ou 30D e/ou ANO) de prevalência, e um ${ }^{13}$ único analisou os três indicadores (7D e 30D e ANO) de prevalência de interesse da presente revisão. 


\begin{tabular}{|c|c|c|c|c|c|c|c|}
\hline \multicolumn{8}{|c|}{$\begin{array}{l}\text { Características dos estudos sobre prevalência de uso de álcool nos últimos sete dias } \\
\text { (7D), } 30 \text { dias (30D) e último ano (ANO) entre estudantes de Medicina }\end{array}$} \\
\hline $\begin{array}{l}\text { Referência/Autor/ } \\
\text { Instituição }\end{array}$ & Ano/Periódico & $\begin{array}{l}\text { Região/ } \\
\text { Local/ } \\
\text { Gestão }\end{array}$ & $\begin{array}{l}\text { Carga } \\
\text { horária }\end{array}$ & $\begin{array}{l}\text { Existência } \\
\text { do curso } \\
\text { (em anos) }\end{array}$ & $\begin{array}{l}\text { Principais } \\
\text { resultados }\end{array}$ & escore & Recomendações \\
\hline $\begin{array}{l}\text { 10. Ferraz et al./ } \\
\text { Unichapecó }\end{array}$ & $\begin{array}{l}2017 \text { / Rev Bras } \\
\text { Promoç Saúde }\end{array}$ & $\begin{array}{l}\text { Sul/ } \\
\text { Interior/ } \\
\text { Privada }\end{array}$ & 7.880 & 6 & $\begin{array}{l}\text { Uso nos } 7 \mathrm{D}= \\
46,5 \% \\
\text { Uso nos } 30 \mathrm{D}= \\
20,2 \%\end{array}$ & 3 & Promover discussões \\
\hline $\begin{array}{l}\text { 11.Petroianu et al. } \\
\text { UFMG }\end{array}$ & $\begin{array}{c}2010 \text { / Rev Assoc } \\
\text { Med Bras }\end{array}$ & $\begin{array}{l}\text { Sudeste/ } \\
\text { Capital/ } \\
\text { Pública }\end{array}$ & 6.660 & 97 & $\begin{array}{c}\text { Uso nos } 7 \mathrm{D}= \\
37,7 \% \\
\text { Uso no } \mathrm{ANO}= \\
85,2 \%\end{array}$ & 3 & Nenhuma \\
\hline $\begin{array}{l}\text { 12. Carvalho et al./ } \\
\text { Santa Casa de SP }\end{array}$ & $\begin{array}{l}2008 \text { / Int J } \\
\text { Adolesc Med } \\
\text { Health }\end{array}$ & $\begin{array}{l}\text { Sudeste/ } \\
\text { Capital/ } \\
\text { Privada }\end{array}$ & 10.052 & 42 & $\begin{array}{c}\text { Uso nos 7D = } \\
35,4 \% \\
\text { Uso nos 30D = } \\
25,5 \%\end{array}$ & 4 & Nenhuma \\
\hline $\begin{array}{l}\text { 13. Kerr-Correa et al./ } \\
\text { Unesp Botucatu }\end{array}$ & $\begin{array}{l}1999 \text { / Rev Bras } \\
\text { Psiquiatr }\end{array}$ & $\begin{array}{l}\text { Sudeste/ } \\
\text { Interior/ } \\
\text { Pública }\end{array}$ & $\mathrm{x}$ & 31 & $\begin{array}{c}\text { Uso nos } 7 \mathrm{D}= \\
23,0 \% \\
\text { Uso nos } 30 \mathrm{D}= \\
50,0 \% \\
\text { Uso no ANO = } \\
79,35 \%\end{array}$ & 7 & $\begin{array}{l}\text { Formatar políticas; fornecer informação } \\
\text { científica, educação para lidar com } \\
\text { estresse, atividades recreativas e de } \\
\text { relaxamento sem uso de substâncias; } \\
\text { elaborar programas de prevenção }\end{array}$ \\
\hline $\begin{array}{l}\text { 14. Tostes et al./ } \\
\text { FM. de Itajubá }\end{array}$ & $\begin{array}{c}2016 \text { / Rev Ciências } \\
\text { da Saúde }\end{array}$ & $\begin{array}{l}\text { Sudeste/ } \\
\text { Interior/ } \\
\text { Privada }\end{array}$ & 7.555 & 45 & $\begin{array}{c}\text { Uso nos } 30 \mathrm{D}= \\
87,6 \%\end{array}$ & 5 & $\begin{array}{c}\text { Elaborar programas e práticas; } \\
\text { instituições, entidades representativas } \\
\text { dos estudantes e famílias devem se unir } \\
\text { para buscar soluções }\end{array}$ \\
\hline $\begin{array}{l}\text { 15. Calheiros et al./ } \\
\text { Unesc (SC) }\end{array}$ & $\begin{array}{c}2013 \text { / Arq Catarin } \\
\text { Med }\end{array}$ & $\begin{array}{l}\text { Sul/ } \\
\text { Interior/ } \\
\text { Privada }\end{array}$ & 8.568 & 12 & $\begin{array}{c}\text { Uso nos } 30 \mathrm{D}= \\
85,4 \% \\
\text { Uso no ANO = } \\
86,9 \%\end{array}$ & 4 & $\begin{array}{l}\text { Implementar ações de prevenção; treinar } \\
\text { habilidades para lidar com estresse; } \\
\text { detectar precocemente o uso; fornecer } \\
\text { material científico; treinar professores }\end{array}$ \\
\hline $\begin{array}{l}\text { 16. Moraes et al./ } \\
\text { UF do Tocantins }\end{array}$ & $\begin{array}{l}2013 \text { / Arq Med } \\
\text { Hosp Fac Cien Med } \\
\text { Santa Casa SP }\end{array}$ & $\begin{array}{l}\text { Norte/ } \\
\text { Capital/ } \\
\text { Pública }\end{array}$ & 8.610 & 6 & $\begin{array}{c}\text { Uso nos } 30 \mathrm{D}= \\
69,7 \%\end{array}$ & 3 & $\begin{array}{l}\text { Programas de orientação e prevenção, } \\
\text { como o Projeto Viver Bem, da Unesp, } \\
\text { e o Programa Viva Mais, da Unicamp. } \\
\text { Incluir disciplina sobre álcool e drogas e } \\
\text { apoio psicopedagógico }\end{array}$ \\
\hline $\begin{array}{l}\text { 17. Oliveira et al./ } \\
\text { USP (dados 1996) }\end{array}$ & $\begin{array}{l}2009 \text { / Rev Bras } \\
\text { Psiquiatr }\end{array}$ & $\begin{array}{l}\text { Sudeste/ } \\
\text { Capital/ } \\
\text { Pública }\end{array}$ & $\mathrm{x}$ & 84 & $\begin{array}{c}\text { Uso nos } 30 \mathrm{D}= \\
76,3 \% \\
\text { Uso no } \mathrm{ANO}= \\
84,6 \%\end{array}$ & 5 & $\begin{array}{c}\text { Estratégias para reconhecimento precoce } \\
\text { de estudantes em risco de uso; alocar } \\
\text { recursos para tratamento, recuperação e } \\
\text { reabilitação }\end{array}$ \\
\hline $\begin{array}{l}\text { 17. Oliveira et al./ } \\
\text { USP (dados 2001) }\end{array}$ & $\begin{array}{l}2009 \text { / Rev Bras } \\
\text { Psiquiatr }\end{array}$ & $\begin{array}{l}\text { Sudeste/ } \\
\text { Capital/ } \\
\text { Pública }\end{array}$ & $\mathrm{x}$ & 89 & $\begin{array}{c}\text { Uso nos } 30 \mathrm{D}= \\
75,7 \% \\
\text { Uso no ANO }= \\
85,7 \%\end{array}$ & 5 & $\begin{array}{c}\text { Estratégias para reconhecimento precoce } \\
\text { de estudantes em risco de uso; alocar } \\
\text { recursos para tratamento, recuperação e } \\
\text { reabilitação }\end{array}$ \\
\hline $\begin{array}{l}\text { 18. Spinelli et al./ } \\
\text { Fac. Med. Jundiaí }\end{array}$ & $\begin{array}{l}2009 / \\
\text { Perspect } \\
\text { Med }\end{array}$ & $\begin{array}{l}\text { Sudeste/ } \\
\text { Interior/ } \\
\text { Pública }\end{array}$ & 10.310 & 38 & $\begin{array}{c}\text { Uso nos } 30 \mathrm{D}= \\
82,5 \% \\
\text { Uso no } \mathrm{ANO}= \\
91,4 \%\end{array}$ & 6 & $\begin{array}{c}\text { Implementar política de prevenção; } \\
\text { aumentar carga horária de aulas sobre } \\
\text { o tema; oferecer apoio psicológico; } \\
\text { criar mecanismos institucionais para } \\
\text { informação e prevenção }\end{array}$ \\
\hline $\begin{array}{l}\text { 19. Tockus e } \\
\text { Gonçalves/ } \\
\text { Positivo }\end{array}$ & $\begin{array}{c}2008 / \\
\text { J Bras Psiquiatr }\end{array}$ & $\begin{array}{l}\text { Sul/ } \\
\text { Capital / } \\
\text { Privada }\end{array}$ & 8.180 & 3 & $\begin{array}{c}\text { Uso nos } 30 \mathrm{D}= \\
70,4 \%\end{array}$ & 4 & $\begin{array}{l}\text { Reconhecer a influência do ambiente } \\
\text { universitário; tomar medidas preventivas } \\
\text { institucionais; triar o uso na instituição e } \\
\text { inserir programas de prevenção }\end{array}$ \\
\hline $\begin{array}{l}\text { 20. Boniatti et al./ } \\
\text { Univ. Caxias } \\
\text { do Sul }\end{array}$ & $\begin{array}{c}2007 \text { / Drug Alcohol } \\
\text { Rev }\end{array}$ & $\begin{array}{l}\text { Sul/ } \\
\text { Interior/ } \\
\text { Privada }\end{array}$ & 8.010 & 38 & $\begin{array}{c}\text { Uso nos } 30 \mathrm{D}= \\
84,7 \% \\
\text { Uso no ANO }= \\
92,9 \%\end{array}$ & 5 & $\begin{array}{c}\text { Propor programa educacional para } \\
\text { debater e esclarecer o uso de drogas } \\
\text { psicoativas durante o curso de Medicina }\end{array}$ \\
\hline $\begin{array}{l}\text { 21. Di Pietro et al./ } \\
\text { Unifesp }\end{array}$ & 2007 / Addict Behav & $\begin{array}{l}\text { Sudeste/ } \\
\text { Capital/ } \\
\text { Pública }\end{array}$ & 9.900 & 73 & $\begin{array}{c}\text { Uso nos } 30 \mathrm{D}= \\
76,9 \%\end{array}$ & 3 & $\begin{array}{l}\text { Propor intervenções preventivas; } \\
\text { questionar o modelo de ensino no campo } \\
\text { de substâncias psicoativas; implementar } \\
\text { estratégias para redução de risco }\end{array}$ \\
\hline $\begin{array}{l}\text { 22. Pereira et al./ } \\
\text { Ufes }\end{array}$ & $\begin{array}{c}2008 \text { / } \\
\text { J Bras Psiquiatr }\end{array}$ & $\begin{array}{l}\text { Sudeste/ } \\
\text { Capital/ } \\
\text { Pública }\end{array}$ & 8.205 & 46 & $\begin{array}{c}\text { Uso nos } 30 \mathrm{D}= \\
48,2 \% \\
\text { Uso no } \mathrm{ANO}= \\
82,1 \%\end{array}$ & 6 & $\begin{array}{c}\text { Inserir disciplinas que tratem de álcool } \\
\text { e outras drogas; criar programas para } \\
\text { prevenção do uso }\end{array}$ \\
\hline $\begin{array}{l}\text { 23. Souza et al./ } \\
\text { UF do Ceará }\end{array}$ & $\begin{array}{l}1999 \text { / Rev Psiq } \\
\text { Clin }\end{array}$ & $\begin{array}{l}\text { Nordeste/ } \\
\text { Capital/ } \\
\text { Pública }\end{array}$ & 8.736 & 49 & $\begin{array}{l}\text { Uso nos } 30 \mathrm{D}= \\
65,6 \% \\
\text { Uso no } \mathrm{ANO}= \\
79,6 \%\end{array}$ & 6 & Nenhuma \\
\hline
\end{tabular}

x (informação indisponível sobre carga horária do curso no ano da coleta de dados da pesquisa original) 
Avaliação de estudos de prevalência de uso de álcool nos últimos sete dias (7D)

Quatro estudos ${ }^{10-13}$ avaliaram o consumo nos últimos sete dias anteriores à entrevista. Três foram desenvolvidos na Região Sudeste e um na Região Sul, sendo dois de escolas públicas e dois de escolas privadas; e dois localizados em capitais e dois no interior. Devido à não sobreposição dos intervalos de confiança, um ${ }^{13}$ estudo parece indicar realmente magnitudes diferentes das dos demais. Apesar do pequeno número de estudos na categoria de 7D, a dispersão sugere que as estimativas de prevalência de uso de álcool mantêm correlação negativa com a carga horária do curso, bem como com os anos de existência da escola médica (Figura 2).

\section{Figura 2}

Representação gráfica das características dos estudos de prevalência de uso de álcool nos últimos sete dias

Prevalência de uso de álcool nos últimos sete dias e características das escolas médicas

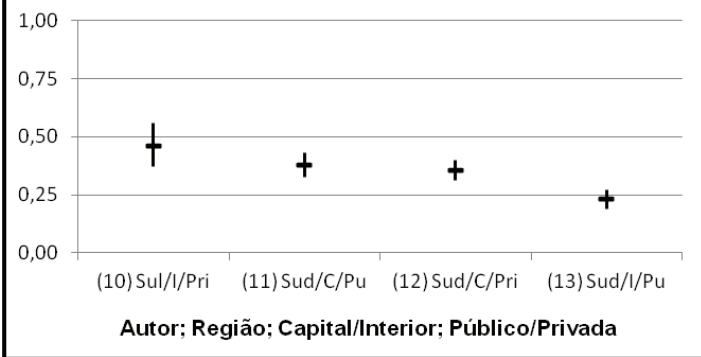

Correlação entre prevalência de uso de álcoo nos últimos sete dias e carga horária do curso de Medicina

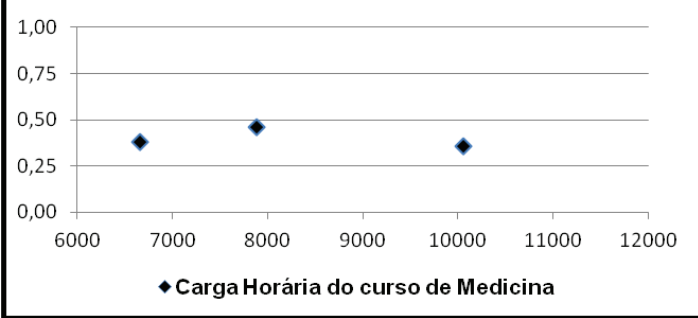

Correlação entre prevalência de uso de álcool nos últimos sete dias e anos de existência da escola médica

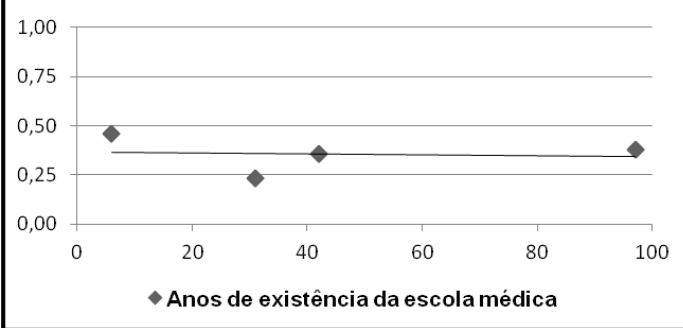

Avaliação de estudos de prevalência de uso de álcool nos últimos 30 dias (30D)

Com exceção de um estudo ${ }^{11}$ com gestão pública e localizado numa capital na Região Sudeste, todos os demais examinaram o indicador de prevalência de 30D. Entre estes últimos, predominou a gestão pública ( $53,8 \%$ versus $46,2 \%)$ e a localização na capital (53,8\% versus $46,2 \%)$. As estimativas de prevalência variaram de $20,2 \%{ }^{10}$ a $87,6 \%{ }^{14}$, com sete co, $15,17-20,21$ estudos mostrando indicadores maiores que $70 \%$. Com respeito à carga horária do curso e aos anos de existência da escola médica, o diagrama de dispersão mostra tendências em direções contrárias, com correlação negativa com a carga horária e positiva com os anos de existência da escola (Figura 3).

\section{Figura 3}

Representação gráfica das características dos estudos de prevalência de uso de álcool nos últimos $\mathbf{3 0}$ dias
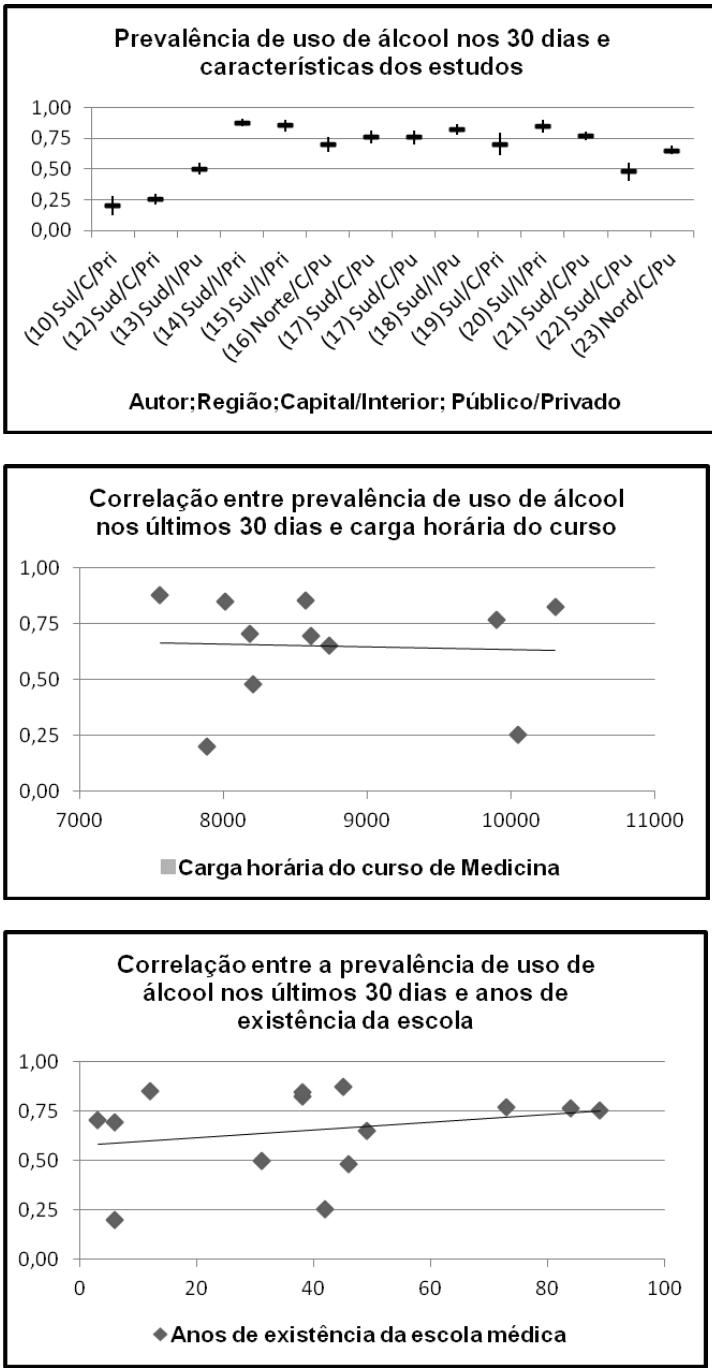
Avaliação de estudos de prevalência de uso de álcool no último ano (ANO)

Oito estudos avaliaram o consumo de álcool no último ANO. Cinco $^{11,13,17,18,22}$ são da Região Sudeste, dois ${ }^{15,20}$ da Sul e um ${ }^{23}$ da Nordeste. Predominou a gestão pública $(75,0 \%$ versus $25,0 \%)$, mas a localização capital/interior teve distribuição similar. As estimativas indicam que o padrão de consumo no último ano pode ser maior que $80 \%$, pois quatro ${ }^{11,13,22,23}$ dos oito estudos nesta categoria tiveram limite inferior dos IC 95\% maior do que 0,800 , e os outros quatro ${ }^{15,17,18,20}$ tiveram IC 95\% maior do que 0,900 . Contudo, devido à completa sobreposição dos limites dos intervalos, não é possível afirmar que o hábito de ingerir álcool seja diferente estatisticamente em alguma das escolas. A verificação de correlação mostra tendência positiva em relação à carga horária e negativa em relação aos anos de existência da escola (Figura 4).

\section{DISCUSSÃO}

Este estudo trouxe como principal contribuição a inclusão das características de cursos e escolas médicas no debate do uso de álcool por estudantes de medicina. A revisão bibliográfica mostrou que a literatura é abundante e aborda diferentes padrões de mensuração. $\mathrm{O}$ interesse em mensurar o consumo de álcool nos últimos sete dias, 30 dias e último ano, apesar de ter sido delimitado em apenas 14 dos estudos incluídos, uniformizou e otimizou as comparações.

Em relação à qualidade dos estudos, questões salientadas

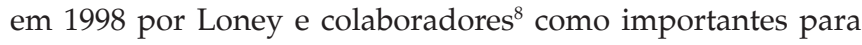
investigações de prevalência não foram plenamente atendidas, pois nenhum dos estudos incluídos obteve escore 8. Chama a atenção que passos metodológicos imprescindíveis, como cálculo de amostra e métodos aleatórios de seleção dos participantes, foram pouco citados. O maior escore (7) foi atribuído ao estudo de Kerr-Correa e colaboradores ${ }^{13}$. Contudo, apenas Oliveira e colaboradores ${ }^{17}$ apresentaram, além das estimativas pontuais de prevalência, os intervalos de confiança de $95 \%$.

Tivemos estudos de instituições de gestão pública e gestão privada, localizadas na capital e também no interior. Considerando as limitações impostas por análises baseadas em aspectos individuais, alguns autores ${ }^{24}$ enfatizam a importância de ampliar as investigações para compreender a influência das instituições propriamente ditas no hábito de ingerir álcool. Contudo, acrescentam que, mesmo utilizando técnicas estatísticas sofisticadas, as evidências ainda são insuficientes para consistentemente explicar o papel de algumas características da escola e do modelo de gestão sobre o uso de álcool ${ }^{24}$.

Não identificamos estudos procedentes da Região Centro-Oeste que atendessem os critérios da presente revisão. Ade-
Figura 4

Representação gráfica das características dos estudos de prevalência de uso de álcool no último ano
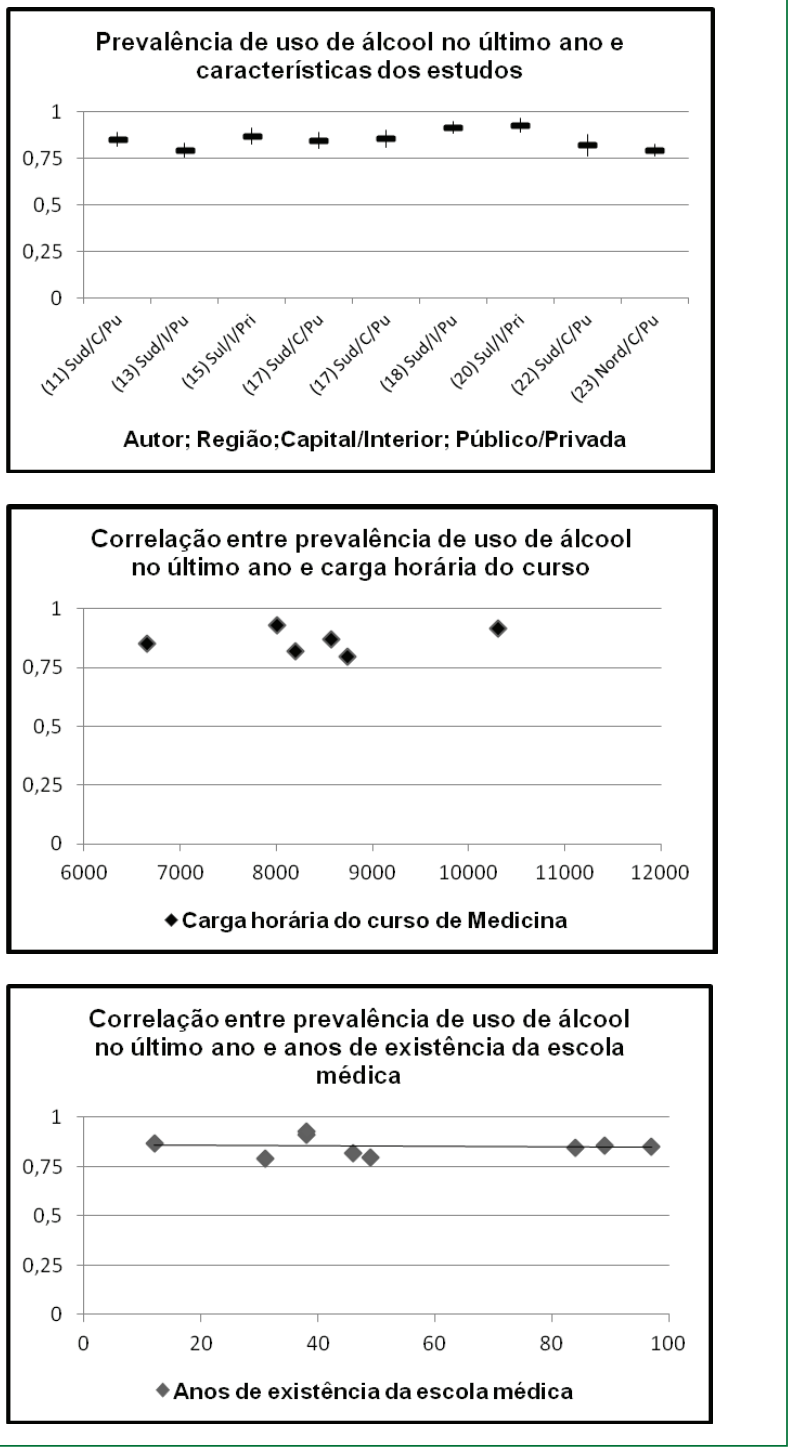

mais, o único estudo ${ }^{23}$ incluído procedente da Região Nordeste examinou o uso de álcool na vida $(92,0 \%)$, no último ano $(79,6 \%)$ e no último mês $(65,5 \%)$, mas teve coleta de dados em 1997. É importante ressaltar que, nos últimos anos, a Região Nordeste foi agraciada com um número razoável de autorizações para abertura de cursos de Medicina9 ${ }^{9}$ Escolas recém-inauguradas supostamente seguem métodos de ensino ativos do tipo Problem-Based Learning (PBL) e contextualizados às necessidades de populações adscritas aos estabelecimentos de ensino e às demandas do Sistema Único de Saúde (SUS). Em comparação com as escolas que adotam métodos tradicionais, 
estruturas recém-inauguradas podem constituir ambientes mais atrativos, com repercussão positiva no bem-estar de estudantes de Medicina, o que se reflete, por exemplo, no menor consumo de substâncias psicoativas, sendo, portanto, merecedoras de mais avaliações científicas.

A magnitude dos indicadores obtidos nesta revisão sugere que a situação de estudantes de Medicina pode ser mais complexa que a de outros grupos populacionais. O padrão de uso no último ano tem as prevalências mais altas, todas acima dos limites estimados em levantamento nacional ${ }^{3}$, tanto para universitários (72\%), quanto para a população geral $(49,8 \%)$. Valores superiores a $90 \%$ foram encontrados por Boniatti e colaboradore ${ }^{20}$ em escola privada do Sul do País e por Spinelli e colaboradores ${ }^{18}$ em instituição pública da Região Sudeste.

Com respeito ao padrão de uso nos últimos 30 dias, a situação foi mais divergente, pois, dos 13 estudos que avaliaram esse consumo, cinco $^{10,12,13,22,23}$ tiveram valores abaixo dos relatados no levantamento nacional ${ }^{3}$, onde as prevalências foram de $60,5 \%$ e de $38,3 \%$, entre universitários e população geral, respectivamente. Nesta revisão, as menores taxas de consumo nos últimos 30 dias ficaram abaixo de $30 \%$ e são de duas escolas privadas ${ }^{10,12}$, localizadas em capitais.

Por considerar que aspectos ambientais também são responsáveis pelo mau uso do álcool nos Estados Unidos, a força-tarefa coordenada pelo National Institute on Alcohol Abuse and Alcoholism (NIAAA) tem induzido intervenções para conter o problema que envolve o campus universitário e comunidades vizinhas ${ }^{25}$. Embora os aspectos ambientais ainda suscitem controvérsias, o tipo de residência usada pelos alunos, o tamanho da instituição e a disponibilidade do produto são reconhecidos como os principais facilitadores. Do ponto de vista individual, as iniciativas mais bem-sucedidas destacam as habilidades cognitivas e comportamentais como merecedoras de mais atenção, com enfoque nas técnicas motivacionais e no trabalho sobre as expectativas dos estudantes com o uso do álcool ${ }^{26}$.

No Brasil, o diagnóstico da situação do uso de álcool vivenciada na Universidade Federal de Santa Catarina gerou proposições que abrangem mudanças curriculares, abertura de espaços oficiais e extraoficiais destinados à discussão sobre o tema, incluindo o desenvolvimento de campanhas preventivas e combate ao uso de substâncias em nível local e nacional ${ }^{27}$. Em 11 dos 14 estudos analisados na presente revisão, os autores se preocuparam em formular algumas recomendações. As propostas em geral mostram que a universidade não pode continuar a se omitir do enfrentamento do problema do álcool. Entre outras ideias, os autores destacaram a importância de formular políticas e criar programas, questionar modelos de ensino, inserir disciplinas sobre o tema, capacitar professores, oferecer materiais, cuidar dos estudantes que fazem uso, incluindo oferta de atividades recreativas e de relaxamento que não façam uso de substâncias, etc. Uma interrogação que permanece, embora não tenha sido objeto desta revisão, é como as universidades brasileiras, em especial as escolas médicas, estão lidando com o problema do álcool em seus estudantes? Houve avanços ou ainda estão apenas no plano da proibição nos campus e ambientes vizinhos?

Com respeito às análises de correlações, primeiramente destacamos a importância dos resultados relativos aos últimos sete dias. Apesar de contarmos com apenas quatro estudos, o padrão de 7D tem a vantagem de conter menor possibilidade de viés de memória e, por conseguinte, de erro de mensuração. Na falta de informações mais precisas sobre frequência e volume ingerido, a análise do padrão de uso em período de tempo mais curto e recente (7D) delineou tendência de prevalências em direção negativa tanto com a carga horária quanto com os anos de existência da escola. Por outro lado, as correlações dos padrões de 30D e um ano envolveram um número maior de estudos, com estimativas de uso de álcool mais elevadas, mas um panorama de maior imprecisão, com as linhas de tendências seguindo em direções contrárias. Em que pese o caráter exploratório do presente estudo, os resultados encontrados, alinhados à gravidade do problema do uso do álcool, justificam a implementação de pesquisas delineadas especificamente para determinar o efeito de componentes ambientais relacionados às escolas e cursos sobre o uso de substâncias, especialmente o álcool, entre os estudantes de Medicina.

Uma das vantagens da atual revisão foi não impor restrições com respeito a idioma e ano de publicação. Contudo, as reformulações implementadas por algumas escolas médicas ao longo dos anos e a falta de informação clara impuseram algumas limitações que inviabilizaram a análise de correlação de carga horária de todas as escolas incluídas. Outra limitação é que, por se tratar de uma revisão sistemática que preencheu todos os passos exigidos por esse tipo de pesquisa, a não implementação de metanálise impediu a estimar uma medida sumária de prevalência de uso de álcool entre estudantes de Medicina.

Concluindo, nossa revisão mostrou que o uso de álcool é tema recorrente na literatura científica no Brasil e que as prevalências nos últimos sete dias, 30 dias e último ano são altas entre estudantes de Medicina. Acentuamos que a influência das características de escolas médicas e de cursos de Medicina sobre o uso de substâncias psicoativas carece de mais atenção da comunidade científica, sendo notória a necessidade do envolvimento das instituições de ensino superior no controle do problema do álcool no Brasil. 


\section{REFERÊNCIAS}

1. World Health Organization. Global status report on alcohol and health. Geneva, WHO Press, 2014.

2. Ministério da Saúde. Secretaria de Vigilância em Saúde. Departamento de Vigilância de Doenças e Agravos não Transmissíveis e Promoção da Saúde. VIGITEL BRASIL 2017 - Vigilância de fatores de risco e proteção para doenças crônicas por inquérito telefônico. Brasília, DF: Ministério da Saúde, 2018.

3. Oliveira LG, Galduróz JCF, Wagner GA, Andrade AG. Uso de drogas pelos universitários brasileiros: contexto nacional e internacional [Capítulo 8]. In: Brasil. Presidência da República. Secretaria Nacional de Políticas sobre Drogas. I Levantamento nacional sobre o uso de álcool, tabaco e outras drogas entre universitários das 27 capitais brasileiras; GREA/IPQ-HC/FMUSP. [organizadores: Andrade AG, Duarte PCAV, Oliveira LG]. Brasília: SENAD, 2010.

4. Peuker AC, Fogaça J, Bizarro L. Expectativas e beber problemático entre universitários. Psic Teor e Pesq 2006; 22(2): 193-200.

5. Borini P, Oliveira CM, Martins MG, Guimarães RC. Padrão de uso de bebidas alcoólicas de estudantes de medicina (Marília, São Paulo): Parte 1. J Bras Psiquiatr 1994; 2(43): 93-103.

6. Fernandes TF, Monteiro BMM, Silva JBM, Oliveira KM, Viana NAO, Gama CAP, et al. Uso de substâncias psicoativas entre universitários brasileiros: perfil epidemiológico, contextos de uso e limitações metodológicas dos estudos. Cad Saude Colet 2017; 25(4): 498-507.

7. Passos SRL, do Brasil PEAA, dos Santos MAB, de Aquino MTC. Prevalence of psychoactive drug use among medical students in Rio de Janeiro. Soc Psychiatry Psychiatr Epidemiol 2006; 41(12): 989-96.

8. Loney PL, Chambers LW, Bennett KJ, Roberts JG, Stratford PW. Critical appraisal of the health research literatura: prevalence or incidence of a health problem. Chronic Dis in Can 1998; 19(4): 170-176.

9. Escolas Médicas do Brasil. Urgente: o ensino médico pede socorro!! + 61 cursos. Disponível em: https://www.escolasmedicas.com.br/news_det.php?cod=2045. Acessado em 18/09/2018.

10. Ferraz L, Rebelatto SL, Schneider GC, Anzolin V. O uso de álcool e tabaco entre acadêmicos de uma universidade do sul do Brasil. Rev Bras Promoç Saúde 2017; 30(1): 79-85.

11. Petroianu A, Reis DCF, Cunha BDS, Souza DM. Prevalência do consumo de álcool, tabaco e entorpecentes por estudantes de medicina da Universidade Federal de Minas Gerais. Rev Assoc Med Bras 2010; 56(5): 568-571.
12. Carvalho KA, Sant'Anna MJ, Coates V, Omar HA. Medical students: abuse of psychoactive substances and sexuality aspects. Int J Adolesc Med Health. 2008; 20(3):321-8

13. Kerr-Corrêa F, Andrade AG, Bassit AZ, Boccuto NMVF. uso de álcool e drogas por estudantes de medicina da UNESP. Rev Bras Psiquiatr 1999; 21(2): 95-100.

14. Tostes JG, Campos FP, Pereira LGR. Consumo de álcool e outras drogas em uma faculdade de medicina do sul de Minas Gerais. Rev Ciências em Saúde 2016; 6(2):

15. Calheiros CS, Brandão LSG, Simões PWTA, Madeira K. Análise do uso de substâncias por estudantes de medicina da Universidade do extremo Sul Catarinense em Criciúma, Santa Catarina. ACM arq catarin med 2013; 42(3):59-63.

16. de Moraes DPA, de Medeiros GMR, Caldas FAXB, Oliveira LA, Baldaçara L. Prevalência do uso de drogas psicotrópicas por estudantes de medicina da Universidade Federal do Tocantins. Arq Med Hosp fac Med Santa Casa São Paulo 2013; 59(3): 127-33.

17. Oliveira LG, Barroso LP, Wagner GA, Ponce JC, Malbergier A, Stempliuk VA, et al. Consumo de drogas entre estudantes de medicina em São Paulo: influências de gênero e ano letivo. Rev Bras Psiquiatr 2009; 31(3): 227-239.

18. Spinelli PA, Valente MFM, Lotério HA. Consumo de álcool e drogas pelos estudantes da Faculdade de Medicina de Jundiaí-SP. Persp Med 2009; 20(2): 19-25.

19. Tockus D, Gonçalves PS. Detecção do uso de drogas de abuso por estudantes de medicina de uma universidade privada. J Bras Psiquiatr 2008; 57(3): 184-187.

20. Boniatti MM, Zubaran C, Panarotto D, Delazeri GJ, Tirello JL, Feldens $\mathrm{MO}$, et al. The use of psychoactive substances among medical students in southern Brazil. Drug Alcohol Rev 2007; 26(3):279-85.

21. Di Pietro MC, Doering-Silveira EB, Oliveira MP, Rosa-Oliveira LQ, Da Silveira DX. Factors associated with the use of solvents and cannabis by medical students. Addict Behav. 2007; 32(8):1740-4.

22. Pereira DS, Souza RS, Buaiz V, Siqueira MM. Uso de substâncias psicoativas entre universitários de medicina da Universidade Federal do Espírito Santo. J Bras Psiquiatr 2008; 57(3): 188-195.

23. Souza FGM, Landim RM, Perdigão FB, Morais RM, Carneiro Filho BA. Consumo de drogas e desempenho acadêmico entre estudantes de medicina no Ceará. Rev Psiquiatr Clin (São Paulo) 1999; 26(4): 188-94.

24. Presley CA, Meilman PW, Leichliter JS. College factors that influence drinking. J Stud Alcohol 2002; Suppl (14): 82-90.

25. Cronce JM, Toomey TL, Lenk K, Nelson TF, Kilmer JR, Larimer ME. NIAAA's College Alcohol intervention matrix. Alcohol Research: current reviews 2017; 39(1): 
26. Iconis R. Understanding alcohol abuse among college students: contributing factors and strategies for intervention. Contemporary Issues in Education Research 2014; 7(3): 243-248

27. Imai FI, Coelho IZ, Bastos JL. Consumo excessivo de álcool, tabagismo e fatores associados em amostra representativa de graduandos da Universidade Federal de Santa Catarina, 2012: estudo transversal. Epidemiol Serv Saúde [online] 2014; 23(3): 435-446.

\section{CONTRIBUIÇÃO DOS AUTORES}

Maria Isabel do Nascimento contribuiu na concepção, planejamento e coordenação da pesquisa, participou da redação do manuscrito e revisão crítica do texto final.

Juliana dos Santos Costa participou do delineamento do estudo, coleta de dados, análise e interpretação dos resultados.

Marcos André Pereira contribuiu na concepção do estudo, coleta de dados e revisão crítica do texto final.

Natália Braga Keher participou da coleta de dados, da análise de dados, apresentação e interpretação dos resultados e redação do manuscrito.

Roberta Fernandes Silva e Moraes contribuiu na coleta de dados, interpretação e apresentação dos resultados e redação do texto.

Todos os autores aprovaram a versão final do manuscrito.

\section{CONFLITO DE INTERESSES}

Os autores afirmam não haver conflito de interesses a declarar. O estudo não recebeu apoio financeiro.

\section{ENDEREÇO PARA CORRESPONDÊNCIA}

Maria Isabel do Nascimento. Rua Marquês de Paraná, 303 prédio principal - segundo andar, Centro, Niterói (RJ), Brasil. CEP 24033-900. Secretaria da Coordenação de Medicina. E-mail: ysamaria@uol.com.br 